\title{
Reliability Aware Task Scheduling In Wireless Hetrogeneous Systems
}

\author{
Sonali T. Bodkhe \\ G.H. Raisoni Academy of Engineering \& \\ Technology, Nagpur, India. \\ Ph.D Scholar at SGB Amravati University
}

\author{
Anjali R. Mahajan, PhD \\ HOD, Department of Information Technology, \\ Government Polytechnic \\ , Nagpur, India.
}

\begin{abstract}
A distributed operating system is one that looks to its user like an ordinary centralized operating system, but runs on multiple, independent nodes. A distributed operating system should:

- control resource allocation to allow their use in the most effective way;

- provide the user with a convenient virtual computer that serves as a high-level programming environment;

- $\quad$ hide the distribution of the resources;

- $\quad$ provide mechanisms for protecting system resources against access by unauthorized users.
\end{abstract}

The main purpose of this research is to explore and use the idle resources and to share the wireless distributed system fairly among the processes. Reliability is taken into account to achieve the goal of scheduling. The comparison study, based on both randomly generated graphs and the graphs of some real applications, shows that proposed scheduling algorithm can achieve reliable task scheduling and improves system reliability significantly. For experimental performance study, a real world application as well as synthetic workloads have been considered. It can be stated that shortening scheduling times improves performance of the system. Hence if scheduling algorithms are applied in parallel to reduce scheduling times, the performance of the heterogeneous clusters will be further enhanced.

\section{General Terms}

Distributed Computing, reliable task scheduling.

\section{Keywords}

Dynamic scheduling, heterogeneous systems, performance, evaluation, processor utilization, reliability, schedulability.

\section{INTRODUCTION}

In heterogeneous cluster computing systems, processor failures and network failures are a common occurrence and can have a negative effect on the applications running on such systems. If failures have to be taken into account effectively, then a reliable scheduling algorithm needs to be employed. However, most of the existing scheduling algorithms for heterogeneous systems consider only common features like scheduling length, and hence do not effectively satisfy the reliability requirements of any task. The problem becomes more prominent in wireless distributed systems where in spite of consideration of various factors, reliability cannot be assured. Therefore a WDS is proposed where some of the parameters of a static distributed system will be considered since scheduling itself is a NP complete problem.

Also reliable scheduling and increase in performance of system cannot be assured at the same time. When it comes to WDS, task scheduling with heterogeneous systems further becomes complex since number of other parameters related to wireless systems have to be taken into account. To present a more realistic and precise scheduling result, scheduling and dispatching times have also been included into the proposed scheduling approach Heterogeneous systems are dependable computing platforms. Many times heterogeneous distributed computing (HDC) systems can achieve higher performance with lesser cost than single super-systems due to resource optimization.

However, in these systems, processors and networks are not free from failure and thus may increase the criticality of the running applications. To deal with such failures, a reliable scheduling algorithm is required. Unfortunately, most scheduling algorithms for scheduling tasks in HDC systems do not sufficiently consider inter-dependent reliability requirements of tasks. Fig. 1 shows a hierarchical structure of task scheduling classifications in distributed systems.

With the use of directed acyclic graph (DAG) a reliabilitybased scheduling algorithm for tasks is proposed, which can achieve a better reliability for applications. A comparative study using both- randomly generated graphs and the graphs of a few real applications, it can be shown that proposed scheduling algorithm gives a better performance over the existing scheduling algorithms in terms of make-span, scheduling length ratio, and reliability[1]. A hierarchical structure of how task scheduling can be classified in distributed systems is shown in Fig.1. At the topmost level, local and global scheduling have been distinguished. Thus local scheduling consists of assigning of processor time of a single processor to multiple processes whereas global scheduling consists of deciding where to execute a process. Therefore local scheduling is left to the local operating system of the processor to which the process is actually allocated. This gives the processors (nodes) increased authority and reduces the responsibility (resulting overhead) of the global scheduling strategy. However it doesn't mean that global scheduling is the job of a single central authority, but rather that the problems of local and global scheduling can be considered as separate issues, and (at least logically) separate mechanisms are required to solve each. The second level in the hierarchy (below global scheduling) provides a choice between static scheduling and dynamic scheduling. This 


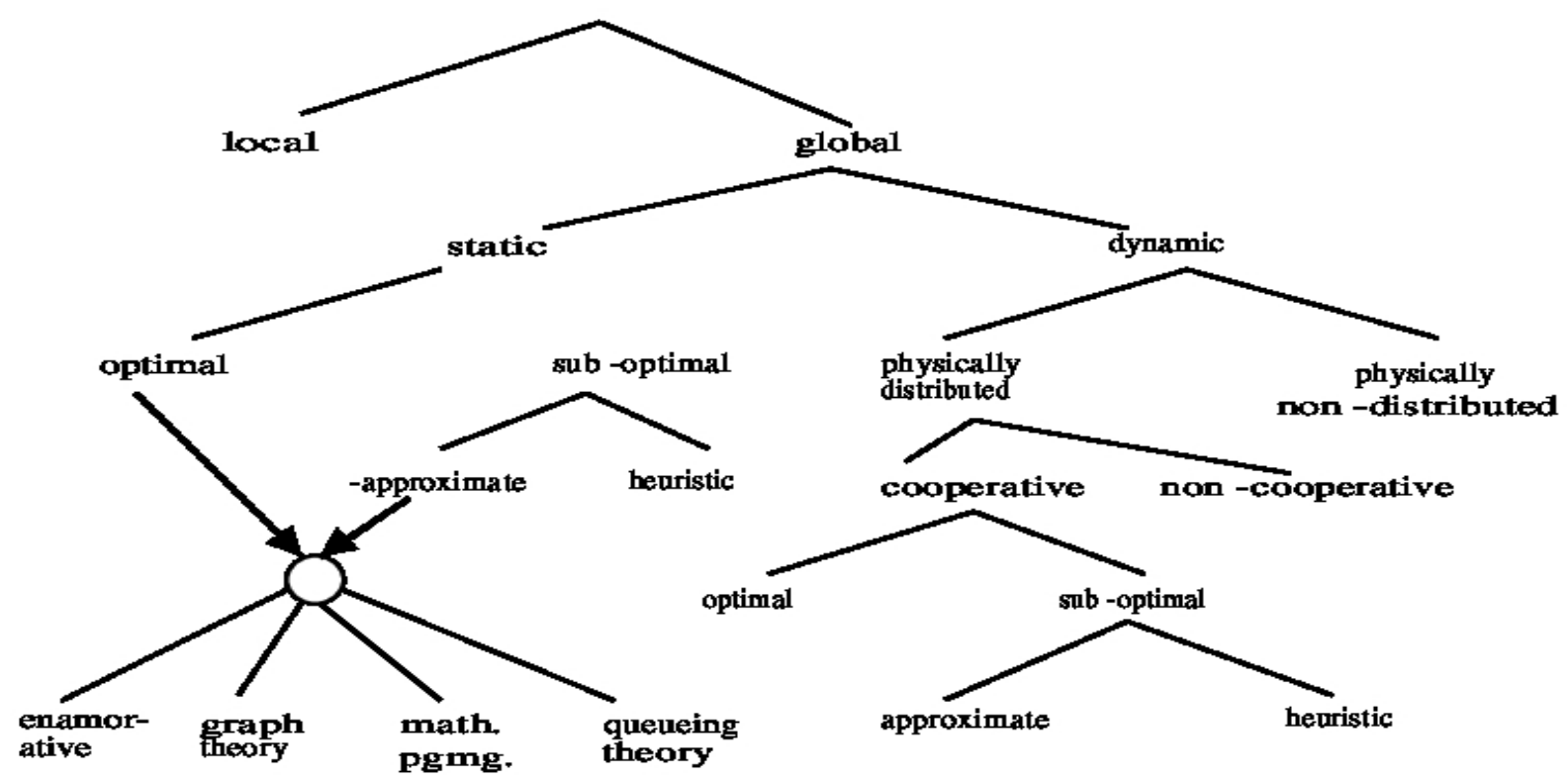

Figure 1: Hierarchical Structure Of Task Scheduling Classifications In Distributed Systems

division is based on the time of making scheduling decisions. Each stochastic task is characterized by its deadline and its time cost distribution, which is represented in terms of mean and variance. Here, static scheduling means assigning of processes to processors before compilation (compile time) whereas dynamic scheduling includes assigning of processes to a processor during execution (run time), and thus can be reassigned while they are running. The other difference is that for static scheduling, decisions are made based only on information regarding the processes and the static system while a dynamic scheduler also takes into account the current state of the system [34].

Wireless Distributed Systems (WDS) are heterogeneous computing systems consisting of a varied set of machines, varied set of communication protocols and programming environments. They offer a range of capabilities in terms of architecture for different execution requirements. One of the challenging areas in such systems is the task scheduling problem that deals with the distribution of a set of limited resources to different tasks to optimize certain performance criterions, like the completion time, communication delay, etc [7][32].

\section{RELATED WORK}

Heterogeneous distributed systems have become widely used for scientific and commercial applications. They are a cluster of normal machines, programmable digital machines, and application oriented ICs [26]. A heterogeneous distributed system involves a number of heterogeneous modules which communicate with each other to solve a problem [27]. The applications running in such systems consist of multiple subtasks that have varied execution requirements. These subtasks need to be ordered for execution and assigned to the various machines in the DS in such a way that the overall execution time is reduced [14].

Nowadays, distributed systems are also being employed in real-time applications, where the systems depend not only on results of computation, but also on the instant at which these results are available. The results of missing deadlines of hard real-time systems may be disastrous, though such consequences for soft real-time systems are comparatively less risky. Some of the hard real-time applications include aircraft control, radar for tracking missiles, and medical electronics, railway crossings, etc. On-line transaction processing systems are soft real-time applications. When it comes to real-time applications, reliability plays an important role and is one of the most important issues. With growing needs of creating reliable real-time applications along with the added advantage of high-speed networks and highperformance machines, wireless distributed systems are being increasingly used for many real-time applications in which the output of the systems depend not only on the results of a computation but also on the time at which these results are produced.

A systematic execution of applications in such environments requires good scheduling strategies that take into account both logical and architectural features to achieve a good assignment of tasks to processors so as to maximize some performance criterion.

Also most of the jobs executed in many real-time systems are critical in nature and therefore high reliability becomes a compulsory requirement of these systems, and the case is especially true for hard real-time applications.

Scheduling plays an important role in obtaining high reliability and performance in distributed computing. Moreover, a task may have different execution times for different inputs. The objective of scheduling is to map tasks onto machines and order their execution so that task precedence requisites are satisfied with a minimum schedule length. Apart from this aim, dynamic scheduling also provides high reliability for non-preemptive, a-periodic, real-time jobs without any additional hardware cost. Here, a framework has been developed that is useful for simulated as well as realtime scheduling by which the jobs are scheduled dynamically, as and when they arrive in a distributed system. In this framework, an identified machine, called scheduler, is made responsible for initiating tasks for execution. The proposed methodology also takes into account the dispatching and scheduling times in addition to reliability costs. Most scheduling algorithms neglect these parameters when dealing with real-time computing. This approach is used in the 
simulation studies to make real-time jobs more predictable and reliable, and the scheduling more realistic.

A lot of scheduling algorithms have been applied earlier in the literature to support real-time scheduling. RT scheduling algorithms are classified into two categories: static or off-line scheduling [1][12][16][18][20][22][25] and dynamic or real time scheduling [10][13][15][17][24][29]. Palis addressed task-scheduling problems with the context of reservationbased real-time systems that guarantee quality of service. The real-time tasks considered in Palis's scheduling framework are preemptive tasks [17], while here it is assumed in proposed scheduling model that real-time tasks are nonpreemptive. Various algorithms mentioned in [16][29] were designed for independent real-time tasks. However the algorithms like those described in [12][20][22], could not schedule tasks with precedence constraints, whereas proposed algorithm which represents tasks by directed acyclic graphs (DAG) can do so. The first testing of the algorithm has been done on non-real-time DAGs and will further be extended it with real-time DAGs to study the real-time scheduling of tasks [18]. However, most of the real-time scheduling algorithms have considered homogeneous systems as the base for implementation, making them unsuitable for use in heterogeneous systems.

In the literature, almost all parallel jobs have been represented by Directed Acyclic Graphs [2][7][12]. Wu et al. in his work, proposed a runtime parallel incremental DAG scheduling approach whereas Cosnard et al. presented a parameterized DAG scheduling algorithm, which first extracts symbolic linear clusters and then assigns these task clusters to various machines [7]. In distributed computing, a typical fork-join paradigm model [23] is used where the main program runs on one processor and spawns a number of tasks from time-totime. Sahni and Vairaktarakis used this paradigm and developed efficient heuristics to obtain reduced finish time schedules [23]. However the scheduling algorithms here were also designed for homogeneous systems only.

Task scheduling in wireless distributed systems with heterogeneous machines has a number of challenges. To name a few, load balancing resource management [8] and reliable scheduling [3][6][31]. Scheduling with heterogeneous systems has been referred in many papers [4][6][11][21][28][30]. It can be found that minimizing the earlier task's completion time further leads to a minimal start time of the next task [14][30]. Topcuoglu et al. in his work referred two efficient as well as low-complexity heuristics for DAGs: the heterogeneous Earliest-Finish-Time (HEFT) algorithm and the other one, the Critical-Path-on-a-Machine (CPOP) algorithm [30]. Özgüner proposed a matching and scheduling framework [9] whereas Maheswaran and Siegel designed a real-time matching and scheduling algorithm for heterogeneous system [14]. Beaumont worked with a static scheduling algorithm but for heterogeneous workstations.

In order that reliability of different resources be also given importance in a system while making scheduling decisions, Ozguner introduced two cost functions that were included into a matching and scheduling algorithm for tasks with precedence constraints [6]. Unfortunately, all the scheduling algorithms assumed that tasks are non-real-time. Hence nonreal-time scheduling algorithms are inefficient in scheduling real-time jobs efficiently, because they are not designed to be capable enough to meet the predictability requirement of realtime jobs.
A lot of work has been done in real-time computing with heterogeneous systems [8][22][24][31]. Tracy et al. considered RT scheduling in distributed systems [31]. Huh, Welch, Shirazi et al. designed an approach for dynamic resource management in real-time heterogeneous systems [8]. Ranaweera and Agrawal worked on scheduling scheme to reduce the number of pipeline stages [22]. Though the above algorithms considered both the real-time and heterogeneous systems issues into consideration, they did not focus on the issue of reliability. The uncertain nature of the task execution times and data transfer rates is also neglected by most traditional scheduling heuristics. Here, real-time scheduling has been proposed in heterogeneous distributed systems, to minimize the reliability cost of the systems. The scheduling algorithms developed in [18] were static in nature, whereas in [this paper], the algorithms were dynamic.

Considerations for scheduling and dispatching times have been ignored by most dynamic algorithms whether they belong to non-real-time or real-time scheduling. To have reliable real-time scheduling results more precise, scheduling and dispatching times need to be incorporated in scheduling algorithms.

In this paper, only few of the parameters have been focused that are necessary for reliable scheduling for real-time systems. Further development in the work will focus on communication link failure and available processor power as the main parameters for reliable scheduling in wireless distributed systems. Hence, a variety of scheduling strategies with reference to non-real-time applications have not been discussed

\section{PROPOSED SYSTEM AND R- MODEL}

Here a generalized system model for parallel applications running on a heterogeneous distributed system is discribed. The various parameters that affect the reliability cost in the reliability model are also discussed in this paper. Computation time, communication delay, etc. are also important for reliable scheduling of real-time applications in wireless heterogeneous systems.

\section{A. System Model}

The following figure-fig. 2 shows the scheduler model commonly used in scheduling processes. The model is similar to the one described in [10][11][15][28], wherein a global scheduler works in association with a Resource Manager.

The tasks are randomly generated using a graph representation language. A schedule queue $(S Q)$ for arriving jobs is maintained by the scheduler According to the dependency of tasks in a job, Directed Acyclic Graphs are constructed. Depending on the precedence constraints of jobs, they are accordingly sorted. The scheduler schedules real-time tasks of each job in SQ and places an accepted job in a dispatch queue $(D Q)$. Using the information conveyed through these DAGs, the tasks are scheduled onto different processors. Thus from the dispatch queue, tasks of each accepted job are transmitted to designated machines, also called processing elements $(P E s)$, for execution. Assuming there are $n$ tasks and $m$ processors an $n * m$ matrix is generated showing the execution costs of available tasks on the $m$ processors. 
Local Queue(LQ)

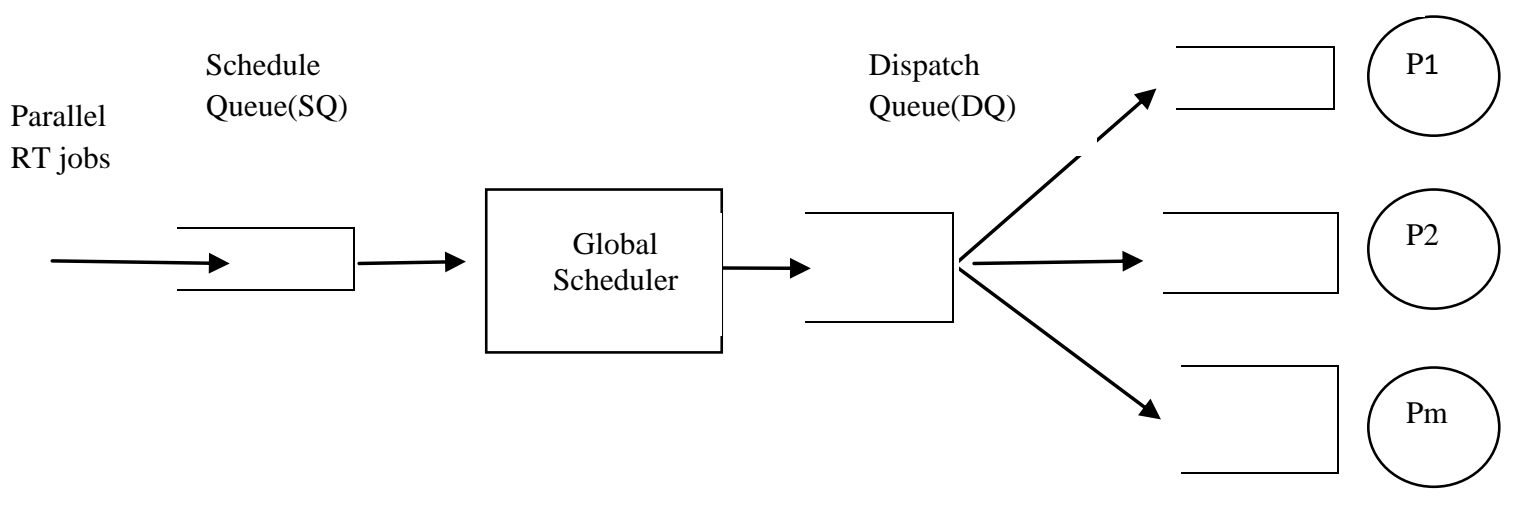

Figure 2: Basic Task Scheduling Model

The scheduler runs in parallel with processors and is not responsible for execution of tasks. Each scheduler maintains its own local queue $(L Q)$ where real-time tasks are transmitted from Dispatch Queue. If all the tasks of an incoming job can be scheduled and completed within deadlines, then the job is considered as acceptable else, the job is rejected.

With distributed scheduling, jobs arrive continuously at each local scheduler and this scheduler produces schedules in parallel with other schedulers. The centralized scheduling scheme however has better approach as compared to distributed scheme. The implementation of a centralized scheduling model is simple and easier than that of a distributed scheduling model. Reliability requires fault tolerance which can be easily provided by the use of a backup scheduler that executes in parallel with the main scheduler. The backup scheduler works independently for identification of schedulable jobs but does not dispatch them to the processors until a failure of the primary scheduler is detected. The disadvantage of using dedicated schedulers is that they remain unutilized, but if when the scheduler is used as a scheduler and processor both, then a prediction mechanism will be required to identify the idle slot of the scheduler. Therefore, the centralized scheduler has been used as the scheduler model.

A real-time job is represented by a directed acyclic graph $(D A G) \mathrm{G}=\{V, E\}$, where $V=\left\{v_{1}, v_{2}, \ldots, v_{n}\right\}$ indicates a set of real-time tasks, and $E$ represents a set of directed edges with weights. The weights indicate the amount of data transmitted among real-time tasks through the edge connecting them. If $e_{i j}$ $=\left(\mathrm{v}_{\mathrm{i}} \mathrm{v}_{\mathrm{j}}\right) \in \mathrm{E}$, then $\mathrm{e}_{\mathrm{ij}}$ denotes a message transmitted from task $v_{i}$ to $v_{j}$, and $\left|e_{i j}\right|$ is the amount of data transmitted between these tasks.

In the proposed model, all tasks arrive from a task generator through a general task queue $(\mathrm{Q})$ and get fed into a central processor called the system processor or the scheduler from where they are distributed to the dispatch queues, Q1,Q2 ,....., Qm , for all of the available processors in the system for execution, as shown in Figure 1.The wireless heterogeneous system is represented by a set $P=\left\{p_{1}, p_{2}, \ldots, p_{m}\right\}$ of machines, where $p$ is a processor with its own memory. Machines in the wireless distributed system communicate with each other through message passing and are interconnected by a high-speed network. The communication delay between two tasks assigned to the same machine is assumed to be zero [19][20] [30].

\section{B. Reliability Model}

The underlying model for computing reliability of WDS is similar as the one defined in [18][19][27], that presumes that permanent failures occur according to a Poisson probability distribution and that the failures are mutually independent. Let $X$ be an $m$ by $n$ binary matrix corresponding to a schedule, where $n$ tasks of a job are assigned to $m$ processors in the system. The entry for element $x$ equals 1 if and only if $v$ has been assigned to $p j$ else $x i j=0$.

Machine failures during an idle time, are not considered since machine's failure during an idle period can be overcome by replacing the failed machine with a spare unit, in proposed reliability model[18][27]. However power required for computation of a task with a wireless distributed system is required to be taken into account [9]. The reliability cost of a task $t_{i}$ on $p_{j}$ is a product of $p_{j}$ 's failure rate $\lambda_{j}$ and $t_{i}$ 's execution time on $p_{j}$. Thus, the total reliability cost of an individual machine is the summation of the reliability costs of all tasks assigned to that machine. Given a vector of failure rates $\Lambda=$ $\left(\lambda_{1}, \lambda_{2}, \ldots, \lambda_{m}\right)$, a specific schedule $X$, and a job $J$, the reliability cost of the distributed system is defined as below,

$$
R C_{P N}(\Lambda, X, J)=\sum_{j=1}^{m} \sum_{i=1}^{n}\left(-\lambda_{j} x_{i j} c_{i j}\right)
$$

To achieve better overall reliability, scheduling a task with larger execution time to a more reliable machine is a better choice. However, scheduling in WDS is a NP hard problem and no defined solutions exist to solve it. The methods that have been used can be divided into 2 categories: exact algorithms and approximation algorithms. The exact algorithms commonly use the branch and bound technique and are applicable to small-sized problems. On the other hand, the approximation algorithms derive sub-optimal task allocations within reasonable times. Heuristic and Genetic algorithms (GAs) have also been adopted for solving problems and obtained promising results. GAs (Goldberg, 1989) belongs to a branch of computational intelligence called metaheuristic. The other fact is that exact algorithms search for optimal solutions and are thus computationally intensive, while metaheuristic algorithms giving near-optimal solutions within reasonable times are more suitable for real-time applications [33]. 


\section{EXPERIMENTS \& RESULTS}

It is assumed that all of these tasks are synchronous, i.e. their first request arrives simultaneously at the time zero. The scheduler will run in parallel with the applications processors, scheduling the new ready tasks, from the task queue (Q), and periodically updating the dispatch queues. This organization ensures that the processors will always find some tasks in the dispatch queues [33][34][35] when they finished their current tasks. The objective of proposed work is to present a simple heuristic scheduling algorithm which accounts for maximizing both the reliability of the application and yielding the required probability for each task in this application to meet its deadline, at the same time. This algorithm exploits parallelism in tasks whenever needed to satisfy the required objectives and improve the previous results.[36].

\section{A. Algorithm:}

\section{\{}

Till there are tasks in the queue

Send them one by one to the central processor or scheduler

\section{Processor $=1$}

While scheduler not empty

Check for each processor in the system

While (processor load $!=\max \& \&$ there is enough processing power)

\{

Assign task to the processor break

\}

Processor $=$ processor +1

If processor $=$ max then processor $=1$

)

One processor is the master processor or scheduler. Following are few of the parameters that were tested for reliability

a. Computation time: time required by each task to execute on each processor.

b. Communication failure rate: the failure rate between two processors during communication.

c. Communication delay: the time required to transfer data between two tasks of two different processors.

d. Intertask communication on same processor is assumed to be zero.

e. Failure rate: probability that the processor will fail.

f. Weighted matrix: amount of data transferred between various communicating tasks.

The graph for CPU utilization is shown in fig. 3 .

The task graph considered is shown in fig. 4 and values for various parameters related to computing are as follows

Failure Rate Matrix:

$\begin{array}{lll}0.000000 & 0.000000 & 0.000000 \\ 0.000000 & 0.000000 & 0.000000 \\ 0.000000 & 0.000000 & 0.000000\end{array}$

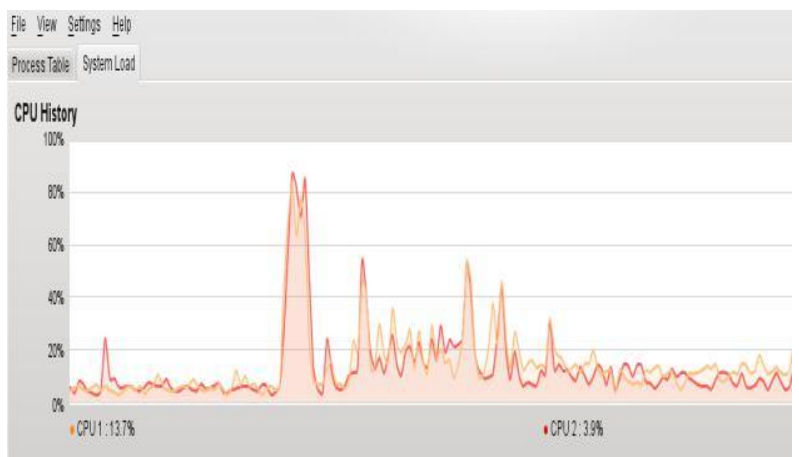

Figure 3: CPU utilization

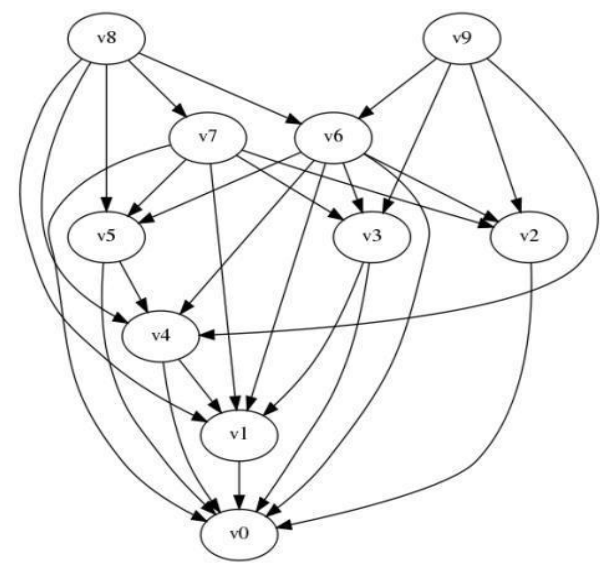

Figure 4. Task graph considered for scheduling

Communication Delay Matrix:

$\begin{array}{lll}0.000006 & 0.000003 & 0.000002 \\ 0.000002 & 0.000005 & 0.000002 \\ 0.000002 & 0.000003 & 0.000003\end{array}$

Computation Time Matrix :

$\begin{array}{lll}0.000000 & 0.000000 & 0.000002 \\ 0.000002 & 0.000003 & 0.000000 \\ 0.000000 & 0.000000 & 0.000006 \\ 0.000003 & 0.000002 & 0.000002 \\ 0.000005 & 0.000002 & 0.000002\end{array}$

Failure Rate Array:

$\begin{array}{lll}0.000000 & 0.000000 & 0.000000\end{array}$

Weighted Matrix

$\begin{array}{lllll}-0.000 & 1.000 & 0.000 & 0.000 & 0.000 \\ -0.000 & 0.000 & 1.000 & 0.000 & 0.000 \\ -0.000 & 0.000 & 0.000 & 3.000 & 0.000 \\ -0.000 & 0.000 & 0.000 & 0.000 & 6.000 \\ -0.000 & 0.000 & 0.000 & 0.000 & 0.000\end{array}$




\section{CONCLUSION}

In this paper, a methodology has been proposed, which maximizes the wireless distributed system reliability for the purpose of successful execution of a task. The scheduler takes care to schedule the tasks onto the appropriate processor so that the task is also completed and there is no overloading of processors. Testing is being carried out for maximizing reliability of scheduling tasks. Once it is done, reliability of real time tasks will also be taken care of for the wireless distributed systems.

\section{REFERENCES}

[1] T. F. Abdelzaher and K.G. Shin, "Combined Task and Message Scheduling in Distributed Real-Time Systems," IEEE Trans. Parallel and Distributed Systems, Vol. 10, No. 11, Nov. 1999.

[2] I. D. Baev, W.M. Meleis, A. Eichenberger, "Lower bounds on precedence-constrained scheduling for parallel machines," Proc. the 29th Int'l Conf. Parallel Processing, pp.549-553, 2000.

[3] O. Beaumont, V. Boudet and Y. Robert, "A Realistic Model and an Efficient Heuristic for Scheduling with Heterogeneous Processors," Proc. 11th Heterogeneous Compting Workshop, 2002.

[4] O. Beaumont, A. Legrand, and Y. Robert, ENS Lyon, L. Carter and J. Ferrante, "Bandwidth-Centric Allocation of Independent Tasks on Heterogeneous Platforms", Proc. Int'l Parallel and Distributed Processing Symp., 2002.

[5] M. Cosnard, E. Jeannot and T. Yang, "SLC: Symbolic Scheduling for Executing Parameterized Task Graphs on Multimachines," Proc. 28th Int'l Conf. Parallel Processing, Fukushima, Japan, 1999.

[6] A. Doğan, F. Özgüner, "Reliable matching and scheduling of precedence-constrained tasks in heterogeneous distributed computing," Proc. Int'l Conf. Parallel Processing, pp. 307-314, 2000.

[7] Xiao Qin , Hong Jiang, "A Dynamic and ReliabilityDriven Scheduling Algorithm for Parallel Real-time Jobs on Heterogeneous Clusters" at Department of Computer Science, New Mexico Institute of Mining and Technology.

[8] E.N. Huh, L.R. Welch, B.A. Shirazi and C.D. Cavanaugh,"Heterogeneous Resource Management for Dynamic Real-Time Systems," Proc. 9th Heterogeneous Computing Workshop, pp. 287-296, 2000.

[9] M. Iverson and F. Özgüner, "Dynamic, Competitive Scheduling of Multiple DAGs in a Distributed Heterogeneous environment," Proc. the 7th Heterogeneous Computing Workshop, pp.70-78, 1998.

[10] V. Kalogeraki, P.M. Melliar-Smith, L.E. Moser, "Dynamic scheduling for soft real-time distributed object systems," Proc. IEEE Int'l Symp. Object-Oriented RealTime Distributed Computing, pp.114-116, 2000.

[11] D. Kebbal, E.G Talbi, and J.M Geib, "Building and scheduling parallel adaptive applications in heterogeneous environments," Proc. IEEE Int'l Workshop Cluster Computing, pp.195-201, 1999.

[12] Y. K. Kwok and I. Ahmad, "FASTEST: A Practical Low-Complexity Algorithm for Compile-Time
Assignment of Parallel Programs to Multiprocessors," IEEE Trans. Parallel and Distributed Systems, Vol.10, No. 2, pp. 147-159, Feb. 1999.

[13] T. Lundqvist and P. Stenstrom, "Timing anomalies in dynamically scheduled micromachines," Proc. IEEE Real-Time Systems Symp., pp.12-21, 1999.

[14] M. Maheswaran and H.J. Siegel, "A Dynamic Matching and Scheduling Algorithm for Heterogeneous Computing Systems," Proc. the 7th Heterogeneous Computing Workshop, pp.57-69, 1998.

[15] G. Manimaran and C.S.R Murthy, "An Efficient Dynamic Scheduling Algorithm for Multimachine RealTime Systems," IEEE Trans. Parallel and Distributed System, Vol. 9, No. 3, pp. 312-315, 1998.

[16] J.C. Palencia, and H.M. Gonzalez, "Schedulability analysis for tasks with static and dynamic offsets," Proc. IEEE Real-Time Systems Symp., pp.26-37, 1998.

[17] A. Palis, "Online Real-Time Job Scheduling with Rate of Progress Guarantees," Proc. 6th Int'l Symp. Parallel Architectures, Algorithms, and Networks, Manila, Philippines, pp. 65-70, 2002.

[18] X. Qin, H. Jiang, C.S. Xie, and Z.F. Han, "Reliabilitydriven scheduling for real-time tasks with precedence constraints in heterogeneous distribute systems," Proc. Int'l Conf. Parallel and Distributed Computing and Systems 2000.

[19] X. Qin and H. Jiang, "Dynamic, Reliability-driven Scheduling of Parallel Real-time Jobs in Heterogeneous Systems," Proc. Int'l Conf.Parallel Processing, Valencia, Spain, pp.113-122, 2001.

[20] X. Qin, H. Jiang, D. R. Swanson, "An Efficient Faulttolerant Scheduling Algorithm for Real-time Tasks with Precedence Constraints in Heterogeneous Systems," Proc. Int'l Conf. Parallel Processing,Vancouver, Canada, pp. 360-368, 2002.

[21] Radulescu, A.J.C van Gemund, "Fast and effective task scheduling in heterogeneous systems," Proc. Euromicro Conf. Real-Time Systems, pp.229-238, 2000.

[22] S. Ranaweera, D.P. Agrawal, "Scheduling of Periodic Time Critical Applications for Pipelined Execution on Heterogeneous systems," Proc. Int'l Conf. Parallel Processing, pp. $131-138,2001$.

[23] S. Sahni and G. Vairaktarakis, "Scheduling for distributed computing,"Proc. IEEE Workshop Future Trends of Distributed Computing Systems, pp. 284-289, 1997.

[24] R.M. Santos, J. Santos, and J. Orozco, "Scheduling heterogeneous multimedia servers: different QoS for hard, soft and non real-time clients," Proc. Euromicro Conf. Real-Time Systems, pp.247-253, 2000.

[25] Shirazi, H.Y. Youn, and D. Lorts, "Evaluation of Static Scheduling Heuristics for Real-Time Multiprocessing," Parallel Processing Letters, Vol. 5, No. 4, pp. 599-610, 1995.

[26] G.C.Sih and E.A.Lee, "A Compile-Time Scheduling heuristic for Interconnection-Constrained Heterogeneous Machine Architectures," IEEE Trans. Parallel and Distributed Systems, 4(2), pp.175-187, 1993. 
[27] S. Srinivasan, and N.K. Jha, "Safty and Reliability Driven Task Allocation in Distributed Systems," IEEE Trans. Parallel and Distributed Systems, 10(3), pp. 238251, 1999.

[28] X.Y. Tang, S.T. Chanson, "Optimizing static job scheduling in a network of heterogeneous computers," Proc. Int'l Conf. Parallel Processing, pp. 373-382, 2000.

[29] M.E. Thomadakis and Jyh-Charn Liu, "On the efficient scheduling of non-periodic tasks in hard real-time systems," Proc. IEEE Real-Time Systems Symp., pp.148-151, 1999.

[30] H. Topcuoglu, S. Hariri and M.Y.Wu, "Task Scheduling Algorithms for Heterogeneous Machines," Proc. Heterogeneous Computing Workshop, pp3-14, 1999.

[31] D. B. Tracy, Noemix, H.J. Siegel and A. Maciejewski, "Static Mapping Heuristics for Tasks with Dependencies, Priorities, Deadlines, and Multiple Versions in Heterogeneous Environments," Proc. Int'l Parallel and Distributed Processing Symp., 2002.

[32] Ms. Sonali T. Bodkhe, Dr. Anjali R. Mahajan, "A Proposed Methodology For Task Scheduling In Wireless
Distributed Systems."Proc. International Conference on Advances in Computing and Information Technology, pp 7-12,ICACIT 2014

[33] Peng-Yeng Yin *, Shiuh-Sheng Yu, Pei-Pei Wang, Yi-Te Wang, "Task allocation for maximizing reliability of a distributed system using hybrid particle swarm optimization" in The Journal of Systems and Software(2007) pages-724-735.

[34] He, Zili Shao, Bin Xiao, Qingfeng Zhuge, Edwin Sha, "Reliability Driven Task Scheduling for Heterogeneous Systems".

[35] S.Sarathambekai*, K.Umamaheswari, “ Task Scheduling in Distributed Systems using Discrete Particle Swarm Optimization" in International Journal of Advanced Research in Computer Science and Software Engineering, Volume 4, Issue 2, February 2014

[36] Ehab Y. Abdel Maksoud, "Performance and ReliabilityDriven Scheduling Approach for Efficient Execution of Parallelizable Stochastic Tasks in Heterogeneous Computing Systems" in Int. J. Open Problems Compt Math., Vol. 3, No. 2, June 2010 